\title{
Avaliação Programática do Estudante: Estratégia Institucional para Melhor Cumprir as Funções da Avaliação Educacional
}

\author{
Luiz Ernesto de Almeida Troncon* \\ Departamento de Clínica Médica \\ Faculdade de Medicina de Ribeirão Preto da Universidade de São Paulo \\ * Autor para correspondência: ledatron@fmrp.usp.br
}

\begin{abstract}
RESUMO
Avaliar o estudante consiste em obter informações sobre o seu desempenho em diferentes domínios, tendo em vista que se cumpram três funções principais: tomar decisões sobre o progresso dele no curso (avaliação somativa), fomentar o aprendizado (avaliação formativa) e contribuir para o controle da qualidade do processo educacional (avaliação informativa). A avaliação programática do aluno de graduação constitui forma inovadora de aplicar com maior eficiência os princípios e métodos da avaliação educacional, para que esta realize com maior eficácia suas atribuições. Na avaliação programática, os procedimentos avaliativos são de responsabilidade da instituição, que define um programa de avaliação centralizado, abrangente e uniforme, no qual professores e coordenadores de disciplina desempenhem diferentes papéis. Este artigo discute as principais funções da avaliação do estudante e as dificuldades para cumpri-las, bem como trata das metas e características mais notáveis da avaliação programática. Consideram-se também aspectos relativos à implantação e ao funcionamento de sistemas estruturados para a avaliação programática do estudante de graduação e a evolução e o estado atual dessa nova prática.
\end{abstract}

Palavras-chave: Avaliação Educacional; Avaliação do Estudante; Avaliação dos Processos de Ensino e Aprendizagem.

\begin{abstract}
Student assessment involves processes of obtaining information on student performance in different domains, which aims at fulfilling three main purposes: fostering learning (formative assessment), making decisions relating to student progress (summative assessment) and informing curriculum quality assurance (informative assessment). Programmatic assessment is an innovative way to perform student assessment more efficiently in order to achieve its purposes with increases efficacy. A main feature of programmatic assessment is that obtaining information on student performance is seen as an institutional responsibility, since the school must define a centralized, comprehensive and uniform program of assessment, in which teachers and faculty members will play a number of different roles. This paper discusses the various purposes of student assessment and the main barriers to achieve them, as well as the key objectives and the most remarkable features of programmatic assessment. Aspects related to introducing and maintaining well-structured systems for student programmatic assessment, the evolution of the ideas underpinning this concept and the current status of this approach are also discussed.
\end{abstract}

Keywords: Student Assessment; Formative Assessment; Summative Assessment; Program Evaluation.

\section{Introdução}

Em recente estágio em escola médica de renomada universidade britânica, tomei contato com o conceito emergente de avaliação programática do estudante, uma forma inovadora de aplicar com maior eficiência princípios e métodos já bem estabelecidos no campo da avaliação educacional.

A avaliação programática constitui uma forma de praticar os procedimentos avaliativos visando a cumprir com maior eficácia as funções da avaliação do estudante (SCHUWIRTH \& ASH, 2013; VAN DER VLEUTEN et al., 2015). Como característica marcante, tal processo destaca-se pelo fato de a avaliação do estudante ser de responsabilidade da instituição, e não dos professores ou coordenadores de disciplina. Estes participam das avaliações segundo aquilo que fora estabelecido no programa específico definido por aquela. 
O esclarecimento de tal conceito demanda discorrer sobre as principais funções da avaliação do estudante e as dificuldades para cumpri-las, bem como sobre as metas e características mais notáveis dos programas de avaliação. Completam este artigo considerações sobre a implantação e o funcionamento de sistemas estruturados de avaliação programática, bem como sobre a sua evolução e estado atual.

\section{As Funções da Avaliação do Estudante e as Dificuldades para Cumpri-las}

A avaliação do estudante compreende processos de obtenção de informações sobre o seu desempenho em diferentes domínios, para que se cumpram três funções principais: embasar decisões sobre o seu progresso (avaliação somativa), fomentar o aprendizado (avaliação formativa) e contribuir para o controle da qualidade dos processos educacionais (avaliação informativa) (VAN DER VLEUTEN et al., 2015; VIANNA, 1989).

A avaliação somativa de qualidade implica que sejam empregados métodos apropriados e abrangentes, em diferentes momentos, de modo a que sejam satisfeitos requisitos de validade e fidedignidade, tendo em vista a tomada de medidas justas e defensáveis (NORCINI et al., 2011). A avaliação formativa visa a corrigir deficiências e reforçar o aprendizado, bem como estimular a autoavaliação e a reflexão, com o objetivo de favorecer o desenvolvimento do estudante. Deve ser praticada de modo continuado, na observação do desempenho do aluno, seguida de feedback de boa qualidade. A função informativa, por sua vez, é cumprida quando os seus resultados disponibilizam dados sobre os processos educacionais para própria instituição, bem como para os órgãos responsáveis pela regulação da educação e, em certos cursos, do exercício profissional. Essa função permite que a avaliação do estudante, juntamente com outras informações, sirva à avaliação do processo educacional e conduza a medidas visando ao seu aperfeiçoamento (MUSICK, 2006).

Na maioria dos cursos de graduação, mostra-se improvável que as três referidas funções sejam cumpridas com adequação. As avaliações somativas são habitualmente pontuais, pouco abrangentes e predominantemente centradas na aquisição de conhecimentos, deixando de lado a incorporação de habilidades e competências relevantes. Ademais, raramente há a preocupação de que as condições de emprego dos vários métodos de avaliação garantam validade e fidedignidade. Quanto à avaliação formativa, diversos estudos relatam que a devolução dos resultados de avaliações aos estudantes e, especialmente, a prática do feedback individualizado são muito infrequentes (VELOSKI et al., 2006). Além disso, inexiste, na maioria das instituições, a prática de se avaliar a qualidade das disciplinas ou a eficácia dos currículos dos cursos de graduação a partir dos dados obtidos na análise pormenorizada dos resultados do desempenho do estudante nos procedimentos avaliativos, principalmente os somativos, até porque a aludida insuficiência de validade e fidedignidade dos exames dificulta a interpretação dos achados.

\section{Metas da Avaliação Programática}

As metas de um programa institucional de avaliação do estudante de graduação podem variar de acordo com a cultura e as características da instituição, bem como com a natureza do curso. No entanto, o próprio conceito de avaliação programática implica o estabelecimento de algumas metas permanentes (Quadro 1). Algumas delas visam ao melhor cumprimento das funções da avaliação do aluno e procuram superar as dificuldades habitualmente encontradas. Dentre estas, destacam-se o reforço e o aprimoramento da avaliação formativa do estudante, bem como a melhoria das condições de emprego dos métodos voltados à avaliação somativa e ao aumento de sua abrangência. Considerando a dificuldade de se preencherem os requisitos de validade e fidedignidade nos diferentes exames, as tomadas de decisão próprias da função somativa podem ser complementadas por informações de natureza qualitativa, sobre o desempenho e o comportamento do estudante, fornecidas pelos professores. Outra importante meta da avaliação 


\section{METAS DA AVALIAÇÃO PROGRAMÁTICA}

1. Aprimorar as condições do emprego dos métodos de avaliação, com vistas ao preenchimento dos requisitos mínimos de validade e fidedignidade.

2. Reforçar a prática da avaliação formativa do estudante, aumentando a frequência com que os estudantes recebem comentários construtivos de fontes externas, preferencialmente na forma de feedba$c k$ individualizado, que estimule o desenvolvimento das capacidades de reflexão e de autoavaliação.

3. Ampliar o espectro da avaliação do estudante, de modo que abranja todas as dimensões do aprendizado e da incorporação de habilidades, competências e boas normas de comportamento profissional e permita acompanhar de modo longitudinal o progresso do estudante.

4. Subsidiar a tomada de decisões sobre o progresso do estudante nos momentos de avaliação somativa não só com resultados quantitativos dos exames, mas também com informações de natureza qualitativa provenientes de fontes legítimas.

5. Contribuir para o controle de qualidade das disciplinas e do próprio currículo a partir da análise dos resultados dos procedimentos avaliativos que revelam o desempenho do estudante e o perfil dos conhecimentos, habilidades e competências adquiridas.

Quadro 1 - Algumas possíveis metas da avaliação programática do estudante de graduação.

programática diz respeito ao acompanhamento longitudinal do progresso do discente pelo acúmulo, ao longo do tempo decorrido em todo o curso de graduação, de informações de natureza quantitativa e qualitativa sobre seu desempenho nos diferentes procedimentos avaliativos as quais podem ser utilizadas nas tomadas de decisão subsequentes. Isso permite que possam ser detectadas dificuldades de progresso e desenvolvimento individual que podem desencadear medidas de remediação, bem como o reconhecimento dos estudantes com alto potencial para a excelência, que podem ser apoiados por meio da adoção de medidas pertinentes.

\section{Características dos Programas de Avaliação}

$\mathrm{Na}$ avaliação programática, a instituição deve definir um programa coerente de avaliação continuada do estudante que preveja a realização de procedimentos avaliativos formativos e somativos em diferentes momentos. Esse programa deve ser gerenciado por um órgão central independente ("centro de avaliação"), responsável por cuidar para que os vários métodos de avaliação discente sejam empregados de maneira uniforme nas diversas disciplinas e áreas de formação, nas melhores condições possíveis. A coordenação do curso, bem como os professores e coordenadores das várias unidades curriculares, trabalham em cooperação com o centro de avaliação e participam de formas distintas do programa institucional, desde que haja independência entre essas diferentes instâncias.

Esse modo de funcionar atende a três importantes características, desejáveis nos sistemas de avaliação programática do estudante: institucionalização, centralização e uniformidade. Outras características almejadas são: abrangência, coerência, continuidade e integração. A abrangência é dada pela inclusão de variados tipos de avaliação, com foco em conhecimentos, habilidades, competências e comportamento, em diferentes ocasiões. A coerência define-se pelo alinhamento da avaliação com o currículo. A continuidade caracteriza-se pela obtenção de informações ao longo do tempo, em conformidade com o progresso do estudante em sua formação. A integração das informações fornecidas pelos diferentes métodos, em vários momentos, 
configura-se na gestão institucional centralizada da avaliação, para que esta cumpra da melhor forma as funções somativa, formativa e informativa da avaliação.

\section{Introdução e Funcionamento de Sistemas de Avaliação Programática}

Definida a vontade política de se adotar a avaliação programática, estabelecido o programa institucional e constituído o centro de avaliação, a instituição deve organizar outras instâncias necessárias ao funcionamento do programa: comitês de tomada de decisão e julgamento de recursos, centro de controle de qualidade e núcleo de desenvolvimento docente com vistas à avaliação. $\mathrm{O}$ centro de avaliação deve também dispor de infraestrutura administrativa e de informática para cumprir suas múltiplas atribuições, do que depende o bom funcionamento do programa (Quadro 2).

Ao longo do curso, o estudante deve manter registro diário das suas atividades (logbook) e periodicamente inserir reflexões sobre algumas delas em seu portfólio. Convém que ele se submeta regularmente a entrevistas com o seu tutor pessoal, que o auxilia no desenvolvimento das suas capacidades de autoavaliação e reflexão, revê-lhe o portfólio e discute o significado das suas atividades e ponderações. Ao cursar diferentes disciplinas, deve receber feedback individualizado dos professores sobre o seu desempenho, que também é registrado em seu portfólio. Pode submeter-se a exames organizados no âmbito da disciplina, mas que têm caráter emi-

\section{CENTRO DE AVALIAÇÃO}

$\checkmark$ Supervisão dos procedimentos de avaliação formativa;

$\checkmark$ Organização e execução de exames com finalidade somativa:

- elaboração e manutenção de banco de questões;

- elaboração e manutenção de banco de estações de avaliação prática;

- análise de resultados de exames.

$\checkmark$ Compilação de:

- certificações de tutores e de coordenadores de unidades curriculares;

- relatos sobre comportamento profissional do estudante;

- manifestações de estudantes sobre circunstâncias especiais.

$\checkmark$ Criação e manutenção de banco de informações sobre o desempenho do estudante ao longo do tempo; $\checkmark$ Envio de resultados de exames e outros dados e informações à instância de tomada de decisões do âmbito da avaliação somativa;

$\checkmark$ Comunicação e interação com instâncias responsáveis por:

- controle de qualidade das ações institucionais;

- capacitação e desenvolvimento docente.

$\checkmark$ Comunicação e interação com diferentes partícipes do programa de avaliação.

\section{PROFESSOR}

$\checkmark$ Atuar como avaliador em procedimentos formativos e somativos;

$\checkmark$ Prover feedback efetivo e de boa qualidade aos estudantes;

$\checkmark$ Atuar como tutor e mentor do estudante;

$\checkmark$ Emitir juízos sobre o estudante, para fins de avaliação somativa;

$\checkmark$ Elaborar questões para exames de conhecimentos;

$\checkmark$ Construir estações para exames práticos;

$\checkmark$ Participar de instâncias previstas no programa:

- centro de avaliação;

- comitê de tomada de decisões somativas;

- comissão julgadora de recursos;

- centro de controle de qualidade;

- centro de capacitação e desenvolvimento docente.

Quadro 2 - Algumas atribuições do centro de avaliação e diferentes modos de participação de professores e coordenadores de disciplinas em um programa institucional de avaliação do estudante. 
nentemente formativo, pois é previsto que receba a devolutiva dos seus resultados.

A avaliação somativa consiste de exames de conhecimentos e de habilidades e competências, organizados pelo centro de avaliação, em condições que garantam validade e fidedignidade. Ocorrem conforme periodicidade estabelecida no programa institucional de avaliação. Seus resultados são encaminhados a comitês de tomada de decisão, organizados por série ou etapa do curso, que também recebem dados de natureza qualitativa, oriundos de tutores, professores e coordenadores de disciplinas, sobre aspectos do desempenho e do comportamento do estudante. Além dessas informações, as decisões levam em conta o histórico do estudante, desde seu ingresso no curso, expresso pelos resultados das avaliações anteriores.

O centro de avaliação é também responsável por encaminhar todos os dados disponíveis do conjunto de estudantes ao centro de controle de qualidade, de modo a viabilizar a avaliação informativa. Esta vai subsidiar a tomada de medidas institucionais para o aprimoramento do currículo, das atividades discentes e do próprio programa de avaliação.

Nesse contexto, cabe aos professores e coordenadores de disciplinas o desempenho de uma variedade de papéis (Quadro 2), que também determinam a qualidade do programa de avaliação e do próprio currículo. Para que isso de fato ocorra, é imprescindível a manutenção de atividades permanentes de capacitação e desenvolvimento docente na área educacional

Evolução e Estado Atual do Conceito e da Aplicação da Avaliação Programática do Estudante - à Guisa de uma Conclusão

O conceito de avaliação programática foi lançado há pouco mais de dez anos por educadores da Universidade de Maastricht, Holanda (VAN DER VLEUTEN \& SCHUWIRTH, 2005). Desde então, diversas maneiras de desenvolver a avaliação programática do estudante vêm sendo introduzidas, experimentadas e aperfeiçoadas (SCHUWIRTH \& VAN DER VLEUTEN, 2011, 2012; RICKETTS \& BLIGH, 2011), em diferentes instituições de vários países, sobretudo do Hemisfério Norte, em cursos e programas de treinamento profissional na área da saúde (RICKETTS \& BLIGH, 2011; VAN DER VLEUTEN et al., 2012; DRIESSEN et al., 2012; BOK et al., 2013).

A aplicação da avaliação programática em escolas médicas teve grande impulso no Reino Unido, a partir de 2009, quando o General Medical Council, órgão regulador do exercício profissional da Medicina e das escolas médicas, estabeleceu recomendações para a avaliação do estudante de Medicina similares às características gerais desta abordagem inovadora. Mais recentemente, relatos de experiências em desenvolvimento no Reino Unido e em outros países (RICKETTS \& BLIGH, 2011; VAN DER VLEUTEN et al., 2012; DRIESSEN et al., 2012; BOK et al., 2013) indicam que, apesar das dificuldades previsíveis, a adoção da avaliação programática, em diferentes escolas médicas e programas de treinamento profissional, é percebida como um avanço em termos da qualidade da avaliação.

O reconhecimento da importância e do potencial de aperfeiçoamento dos sistemas de avaliação programática do estudante justificou o estabelecimento de diretrizes voltadas às condições determinantes da excelência no seu funcionamento e da sua sustentabilidade institucional no longo prazo, feitas por um painel internacional de especialistas em avaliação do estudante (DIJKSTRA et al., 2012). Essas diretrizes tratam das medidas envolvendo participantes e instituições, de modo a definir os objetivos e metas do programa, em relação à infraestrutura disponível e os recursos necessários. Recomendações sobre como superar dificuldades na implantação de programas de avaliação tem sido também publicadas.

As características e os modos de funcionamento dos sistemas de avaliação programática do estudante adequam-se muito bem aos cursos de graduação na área da saúde que, além da educação geral e do desenvolvimento pessoal do estudante, visam à incorporação de habilidades, competências e comportamento profissionais bem definidas e passíveis de avaliação acurada. 
É plausível, porém, que a aplicação desta forma inovadora de avaliar o estudante, definida em um programa institucional, possa também ser bem sucedida em outros cursos das diferentes áreas do conhecimento.

\section{Referências Bibliográficas}

BOK, Harold G.; TEUNISSEN, P. W.; FAVIER, R. P.; RIETBROEK, N.J.; THEYSE, L. F.; BROMMER, H.; HAARHUIS, J. C.; VAN BEUKELEN, P.; VAN DER VLEUTEN, C. P. M.; JAARSMA, D. A. "Programmatic Assessment of Competency-based Workplace Learning: When Theory Meets Practice". BMC Med Educ., vol. 13, n. 1, 2013, p. 1.

DIJKSTRA, Joost; GALBRAITH, R.; HODGES, B. D.; MCAVOY, P. A.; MCGRORIE, P.; SOUTHGATE, L. J.; VAN DER VLEUTEN, C. P. M.; WASS, V.; SCHUWIRTH, L. W. "Expert Validation of Fit-for-purpose Guidelines for Designing Programmes of Assessment". BMC Med Educ., vol. 12, n. 1, 2012, p. 20.

DRIESSEN, Erik W.; VAN TARTWIJK, J.; GOVAERTS, M.; TEUNISSEN, P.; VAN DER VLEUTEN C. P. M. "The Use of Programmatic Assessment in the Clinical Workplace: A Maastricht Case Report". Med Teach, vol. 34, n. 3, 2012, pp. 226-31.

MUSICK, David W. "A Conceptual Model for Program Evaluation in Graduate Medical Education". Acad Med, vol. 81, n. 8, 2006, pp. 759-765.

NORCINI, John; ANDERSON, B.; BOLLELA, V.; BURCH, V.; COSTA, M. J.; DUVIVIER, R.; GALBRAITH, R.; HAYS, R.; KENT, A.; PERROTT, V.; ROBERTS, T. "Criteria for Good Assessment: Consensus Statement and Recommendations from the Ottawa 2010 Conference". Med Teach, vol. 33, n. 3, 2011, pp. 206-214.
RICKETTS, Chris \& BLIGH, J. "Developing a 'Frequent Look and Rapid Remediation' Assessment System for a New Medical School. Acad Med. 86, n.1, 2011, pp. 67-71.

SCHUWIRTH, Lambert \& ASH, J. "Assessing Tomorrow's Learner: In Competency-based Education Only a Radically Different Holistic Method of Assessment Will Work: Six Things We Could Forget". Med Teach., vol. 35, n. 7, 2013, pp. 555-560.

SCHUWIRTH, Lambert W. T. \& VAN DER VLEUTEN, C. P. M. "Programmatic Assessment: From Assessment of Learning to Assessment for Learning". Med Teach, vol. 33, n. 6, 2011, pp. 478-485.

"Programmatic Assessment and Kane's Validity Perspective". Med Educ., vol. 46, n. 1, 2012, pp. 38-48.

VANDER VLEUTEN, CeesP.M.;SCHUWIRTH, L. W. T.; DRIESSEN, E. W.; GOVAERTS, M. J. B.; HEENEMAN, S. "Twelve Tips for Programmatic Assessment." Med Teach, vol. 37, n. 7, 2015, pp. 641-646.

VANDER VLEUTEN, Cees P.M.;SCHUWIRTH, L. W.; DRIESSEN, E. W.; DIJKSTRA, J.; TIGELAAR, D.; BAARTMAN, L. K.; VAN TARTWIJK, J. "A Model for Programmatic Assessment Fit for Purpose". Med Teach, vol. 34, n. 3, 2012, pp. 205-214.

VAN DER VLEUTEN, Gees P. M. \& SCHUWIRTH L.W. "Assessing Professional Competence: From Methods to Programmes". Med Educ., vol. 39, n. 3, 2005, pp. 309-317.

VELOSKI, Jon; BOEX, J. R.; GRASBERGER, M. J.; EVANS, A.; WOLFSON, D. B. "Systematic Review of the Literature on Assessment, Feedback and Physicians' Clinical Performance: BEME Guide No. 7". Med Teach, vol. 28, n. 2, 2006, pp. 117-128.

VIANNA, Heraldo Marelin. Introdução à Avaliação Educacional. São Paulo: IBRASA, 1989.

Publicado em 05/07/2016. 